\title{
Strong-coupling Bose polarons in one dimension: Condensate deformation and modified Bogoliubov phonons
}

\author{
J. Jager $\odot,{ }^{1}$ R. Barnett $\odot,{ }^{1}$ M. Will $\odot,{ }^{2}$ and M. Fleischhauer $\odot^{2}$ \\ ${ }^{1}$ Department of Mathematics, Imperial College London, London SW7 2AZ, United Kingdom \\ ${ }^{2}$ Department of Physics and Research Center OPTIMAS, University of Kaiserslautern, 67663 Kaiserslautern, Germany
}

(Received 3 February 2020; accepted 2 July 2020; published 24 July 2020; corrected 22 April 2021)

\begin{abstract}
We discuss the interaction of a quantum impurity with a one-dimensional degenerate Bose gas forming a Bose polaron. In three spatial dimension, the quasiparticle is typically well described by the extended Fröhlich model, in full analogy with the solid-state counterpart. This description, which assumes an undepleted condensate, fails, however, in 1D, where the backaction of the impurity on the condensate leads to a self-bound mean-field polaron for arbitrarily weak impurity-boson interactions. We present a model that takes into account this backaction and describes the impurity-condensate interaction as coupling to phononlike excitations of a deformed condensate. A comparison of polaron energies and masses to diffusion quantum Monte Carlo simulations shows very good agreement already on the level of analytical mean-field solutions and is further improved when taking into account quantum fluctuations.
\end{abstract}

DOI: 10.1103/PhysRevResearch.2.033142

\section{INTRODUCTION}

The polaron, introduced by Landau and Pekar [1,2] to describe the interaction of an electron with lattice vibrations in a solid, is a paradigmatic model of quasiparticle formation in condensed-matter physics. A hallmark feature of the quasiparticle is mass enhancement: the electron becomes dressed by a cloud of phonons which in turn affects its dynamical properties. The polaron concept has wide applications across condensed-matter physics ranging from charge transport in organic semiconductors to high- $T_{c}$ superconductivity $[3,4]$.

More recently, neutral atoms immersed in quantum gases have attracted much attention since they are experimentally accessible platforms for studying polaron physics with high precision and in novel regimes. For example, the impuritybath interaction can be tuned from weak to strong coupling employing Feshbach resonances [5]. In such systems, the impurity atom is immersed in a superfluid and a polaron is formed by its interaction with the collective excitations of the superfluid. The Fermi-polaron, i.e., an impurity in a degenerate Fermi gas, has been studied in a number of experiments [6-14]. In contrast, only a few experiments on Bose polarons exist [15-18]. Due to the compressibility of a Bose gas, a large number of excitations can be generated, and interactions within the Bose gas are important.

Theoretical works addressing the Bose polaron most often describe the interaction with the impurity as a coupling to Bogoliubov phonons of a uniform superfluid [19-24]. The

Published by the American Physical Society under the terms of the Creative Commons Attribution 4.0 International license. Further distribution of this work must maintain attribution to the author(s) and the published article's title, journal citation, and DOI. resulting (extended) Fröhlich Hamiltonian is formally identical to the one used in solid-state systems [25], amended with two-phonon scattering terms. Efficient approaches for its solution beyond the perturbative regime have been developed in the past, including variational [24,26,27], field-theoretical [19,28-30], renormalization group (RG) [23,31], and opensystem approaches [32], as well as quantum Monte-Carlo simulations $[23,33,34]$. However, as well known from the example of electrons in superfluid helium, a strongly interacting impurity can also distort the superfluid itself [35]. This deformation creates a potential for the impurity which can lead to a self-bound state. In 3D, the normalized impurity-Bose interaction has to exceed a critical value for this, given by the inverse gas parameter [36-38]. Since for typical condensates the gas parameter is very small, the extended Fröhlich model remains adequate.

The situation is different in 1D, which was experimentally realized in Ref. [15]. Here an arbitrarily weak deformation of the condensate leads to a self-localized impurity [37]. This restricts the accuracy of the Fröhlich model to the perturbative regime. In fact, a comparison between exact diffusion Monte Carlo (DMC) simulations of the full model with RG solutions of the extended Fröhlich model in Ref. [23] shows that this model is only accurate for weak interactions and breaks down completely for attractive interactions at intermediate interactions.

In this paper, we follow a different approach, and expand the Bose quantum field about the exact mean-field solution in the presence of the mobile impurity in the Lee-LowPines (LLP) frame [39]. Such a treatment incorporates the backaction of the impurity already at the mean-field level as in Refs. [36-38], but keeps the entanglement between impurity and BEC by working in the LLP frame. Quantum effects are then taken into account by the coupling to phononlike excitations of the deformed superfluid. Motivated by 
experiments [15] and the availability of semianalytic meanfield solutions, we here consider a 1D quasi condensate with weak to moderate boson-boson interactions. While the experiments are performed in a harmonic trap, we assume periodic boundary conditions when introducing phonons. Strictly speaking, there is no BEC in a homogeneous 1D system and also the quasiparticle concept is believed to break down $[40,41]$ due to a diverging number of low-energy excitations emitted by the impurity. Thus, special care must be taken when calculating quantum effects. We derive the effective Hamiltonian for the deformed phonons and solve them in the Bogoliubov approximation. Our treatment carries over naturally to higher dimensional systems with the only difference that the mean-field solutions have to be obtained numerically. Other treatments of the 1D polaron based on a factorization of the $N$-particle wave function in the LLP frame exist that take the deformation of the condensate into account [42-45]. The scope of extending them to incorporate quantum fluctuations is limited, however. We note that the standard arguments to define the polaron mass, applicable for Fröhlich-type models, give nonsensical results here and require a careful reconsideration. We derive analytical expressions for the mean-field polaron wave function, from which we reproduce previous approximations for the polaronic mass and energy. We then calculate quantum corrections by solving the Bogoliubov deGenne equations in a self-consistent approach. Our results are benchmarked against recent DMC results [23]. We find very good agreement in all regimes for repulsive interactions underpinning the hypothesis that expanding about the nonuniform condensate is an excellent starting point. We also present results for attractive interactions. Here we find again very good agreement with DMC for the energy of the polaron but less good agreement for the mass. We attribute this discrepancy to the existence of many-particle bound states in the attractive regime $[23,33]$.

\section{MODEL AND PROPER DEFINITION OF POLARON MASS}

Our starting point is a single impurity atom coupled to $N$ identical bosons in one dimension, described by the Hamiltonian

$$
\begin{aligned}
\hat{\mathcal{H}}= & \int d x \hat{\phi}^{\dagger}(x)\left(-\frac{1}{2 m} \partial_{x}^{2}+\frac{g_{\mathrm{BB}}}{2} \hat{\phi}^{\dagger}(x) \hat{\phi}(x)-\mu\right. \\
& \left.+g_{\mathrm{IB}} \delta(x-\hat{X})\right) \hat{\phi}(x)+\frac{\hat{P}^{2}}{2 M} .
\end{aligned}
$$

Here $m(M)$ denotes the mass of the bosons (impurity atom), $\hat{\phi}(x)$ is the Bose field operator, $g_{\mathrm{BB}}\left(g_{\mathrm{IB}}\right)$ are the bosonboson (boson-impurity) interaction strength, $\hat{X}(\hat{P})$ denotes the position (momentum) operator of the impurity, and $\mu$ is the chemical potential of the Bose gas. Throughout the paper, we set $\hbar=1$ and employ periodic boundary conditions of length $L$. The relative interaction strength is denoted by $\eta=$ $g_{\mathrm{IB}} / g_{\mathrm{BB}}$ and we introduce the healing length $\xi=1 / \sqrt{2 m \mu}$ and the speed of sound $c=\sqrt{\mu / m}$. Expanding the bosonic field operator in Eq. (1) around a homogenous condensate as $\hat{\phi}(x)=\sqrt{n_{0}}+\hat{\xi}(x)$ with $n_{0}=N / L$ leads to the extended Fröhlich Hamiltonian [24,25]. In this paper, we choose a different starting point and consider the effects of the impurity already at the level of the condensate.

Before delving into the solutions of the mean-field equations, it is important to point out some fundamental differences between the ground state of the effective Fröhlich and the full Hamiltonian Eq. (1) for finite momentum. For the Fröhlich model, it is easy to show that for fixed total momentum, the ground state is indeed the polaronic solution $[23,39,46]$. The situation is very different for Eq. (1). Indeed, the ground state for finite momentum for this case is the uniformly boosted system. To see this, we introduce the potential $\hat{\Omega}=\hat{\mathcal{H}}-v \hat{P}_{\text {tot }}$, with total momentum $\hat{P}_{\text {tot }}=\hat{P}_{\mathrm{B}}+\hat{P}$ where $\hat{P}_{\mathrm{B}}=-i \int \hat{\phi}^{\dagger}(x) \partial_{x} \hat{\phi}(x) \mathrm{d} x$. It is straightforward to see that finding the constrained ground state of Eq. (1) (with fixed total momentum) is equivalent to finding the unconstrained ground state of $\hat{\Omega}$ for a given $v$ which acts as a Lagrange multiplier. Introducing the unitary transformation $\hat{U}_{\mathrm{cm}}=\exp \left(-i M_{\mathrm{tot}} \hat{X}_{\mathrm{cm}} v\right)$, with $M_{\mathrm{tot}}=N m+M$ and $\hat{X}_{\mathrm{cm}}=$ $\frac{1}{M_{\mathrm{tot}}}\left(m \int \mathrm{d} x x \hat{\phi}^{\dagger}(x) \hat{\phi}(x)+M \hat{X}\right)$ to boost into the center-ofmass frame, one finds $\hat{\Omega}=\hat{U}_{\mathrm{cm}}^{\dagger} \hat{\mathcal{H}} \hat{U}_{\mathrm{cm}}-\frac{1}{2} M_{\mathrm{tot}} v^{2}$. With this expression, one can clearly relate eigenstates of $\hat{\mathcal{H}}$ with those of $\hat{\Omega}$. In particular, the ground state for finite momentum (corresponding to finite $v$ ) is the boosted ground state and the effective mass of the polaron always equals the total mass. Such a uniformly boosted system is precluded in the Fröhlich model.

We proceed as in the case of the Fröhlich model and eliminate the impurity position operator from Eq. (1) by a LLP- [39] type transformation $\hat{U}_{\mathrm{LLP}}=\exp \left(-i \hat{X} \hat{P}_{\mathrm{B}}\right)$. Here, in contrast, the total momentum of the bosons $\hat{P}_{\mathrm{B}}$ enters. $\hat{U}_{\mathrm{LLP}}$ transforms to a comoving frame, where the impurity is at the origin and its momentum is transformed to the conserved total momentum of the system which can be treated as a c-number $P$. By eliminating the impurity from the problem by an exact transformation, entanglement between the impurity and the condensate is already included on the mean-field level and we do not have to assume a factorized wave function as, for example, done in Refs. [37,38]. At the same time, an impurity-mediated interaction between the bosons $\sim \int \mathrm{d} x\left(P-\hat{P}_{\mathrm{B}}\right)^{2} / 2 M$ emerges in the transformed Hamiltonian. To treat this, it will prove helpful to introduce a Hubbard-Stratonovich field $\hat{u}$, which gives

$$
\begin{aligned}
& \hat{\mathcal{H}}_{\mathrm{LLP}}^{\mathrm{S}} \\
& =\int d x \hat{\phi}^{\dagger}(x)\left(-\frac{1}{2 m_{\mathrm{r}}} \partial_{x}^{2}+\frac{g_{\mathrm{BB}}}{2} \hat{\phi}^{\dagger}(x) \hat{\phi}(x)-\mu+g_{\mathrm{IB}} \delta(x)\right) \\
& \quad \times \hat{\phi}(x)+\hat{u}\left(P-\hat{P}_{\mathrm{B}}\right)-\frac{1}{2} M \hat{u}^{2},
\end{aligned}
$$

where $\hat{u}$ satisfies $M \hat{u}=P-\hat{P}_{\mathrm{B}}$, and can thus be viewed as the impurity velocity. $m_{\mathrm{r}}=(M+m) / M m$ is the reduced mass, and we defined rescaled healing length $\bar{\xi}=\sqrt{m / m_{\mathrm{r}}} \xi$ and speed of sound $\bar{c}=\sqrt{m / m_{\mathrm{r}}} c$. 


\section{MEAN-FIELD SOLUTIONS}

\section{A. Mean-field equations in the presence of the impurity}

We now expand $\hat{\phi}(x)=\phi(x)+\hat{\xi}(x)$ and $\hat{u}=u+\delta \hat{u}$, where $\phi(x)$ and $u$ are chosen to solve the mean-field equations of Eq. (2); for details, see Appendix A,

$$
\begin{gathered}
\left(-\frac{1}{2 m_{\mathrm{r}}} \partial_{x}^{2}+g_{\mathrm{BB}}|\phi(x)|^{2}-\mu+i u \partial_{x}\right) \phi(x)=0, \\
\left.\partial_{x} \phi(x)\right|_{0^{-}} ^{0^{+}}=2 m_{\mathrm{r}} g_{\mathrm{IB}} \phi(0),
\end{gathered}
$$

subject to the boundary conditions $\phi\left(\frac{L}{2}\right)=\phi\left(-\frac{L}{2}\right)$ and $|\phi( \pm L / 2)|^{2}=n_{0}+\mathcal{O}(1 / L)$. Note that to remedy the problem of the uniformly boosted system being the ground state, we require that the polaron is a local quantity. Thus the condensate must be stationary far away from the impurity up to $1 / L$ corrections. Solutions of the mean-field equations exist in the literature where the phase is not periodic [42,47] and have been applied to the 1D polaron before [43-45]. The nonperiodic phase corresponds to unphysical sources at the boundary and leads to wrong predictions such as a negative kinematic polaron mass. We instead find the mean-field solution of the form $\phi(x)=\sqrt{n(x)} e^{i \theta(x)}$ (see Appendix A for more details),

$$
n(x)=\frac{\mu}{g_{\mathrm{BB}}}\left(1-\beta \operatorname{sech}^{2}\left(\sqrt{\beta / 2}\left(|x|+x_{0}\right) / \bar{\xi}\right)\right),
$$

with $\beta=1-\frac{u^{2}}{\bar{c}^{2}}+\mathcal{O}\left(1 / L^{2}\right)$ and $\mu=g_{\mathrm{BB}} n_{0}^{\mathrm{MF}}-\left(\partial_{x} \theta_{1}\right) u+$ $\mathcal{O}\left(1 / L^{2}\right)$. If we consider the mean-field solution alone, we fix $n_{0}^{\mathrm{MF}}=n_{0}\left(1+2 \sqrt{2 \beta} \bar{\xi} / L\left(1-\tanh \left(\sqrt{\beta / 2} x_{0} / \bar{\xi}\right)\right)\right)+$ $\mathcal{O}\left(1 / L^{2}\right)$, where $n_{0}=N / L$ is the average density of bosons. Upon considering quantum fluctuations later on, the meanfield density needs to be adjusted. For the phase, we find $\theta(x)=\theta_{0}(x)+(2 f(0)-2 f(L / 2)) x / L$ with

$$
f(x)=\arctan \left(\frac{\sqrt{4 u^{2} \beta / \bar{c}^{2}}}{e^{\sqrt{2 \beta}\left(x+x_{0}\right) / \bar{\xi}}-2 \beta+1}\right)
$$

for $x>0$ and $\theta_{0}(x)=2 f(0)-f(-x)$ for $x<0$. Finally, we determine $x_{0}$ through the jump condition for the derivative. In the limit $u=0$, we find for $g_{\mathrm{IB}}>0: x_{0}=\frac{\bar{\xi}}{\sqrt{2}} \log (y)$, with $y=$ $\sqrt{1+8 \frac{n_{0}^{2} \bar{\xi}^{2}}{\eta^{2}}}+2 \frac{\sqrt{2} n_{0} \bar{\xi}}{\eta}$ and for $g_{\mathrm{IB}}<0$, we have $x_{0} \rightarrow x_{0}^{\mathrm{a}}=$ $x_{0}+i \pi / 2 \bar{\xi}(2 / \beta)^{1 / 2}$. It is instructive to insert $x_{0}^{\text {a into Eq. }}$ (5) and obtain the density profile for the attractive side explicitly:

$$
n^{a}(x)=\frac{\mu}{g_{\mathrm{BB}}}\left(1+\beta \operatorname{csch}^{2}\left(\sqrt{\beta / 2}\left(|x|+x_{0}\right) / \bar{\xi}\right)\right) .
$$

It becomes apparent that the density far away of the impurity now is lowered instead of increased, and is given by $n_{0}^{\mathrm{MFa}}=n_{0}\left(1-2 \sqrt{2 \beta} \bar{\xi} / L\left(\operatorname{coth}\left(\sqrt{\beta / 2} x_{0} / \bar{\xi}\right)-1\right)\right)+$ $\mathcal{O}\left(1 / L^{2}\right)$. This seemingly small correction can have a profound impact for $|\eta| \gg 1$. In this limiting case, $\left(\operatorname{coth}\left(\sqrt{\beta / 2} x_{0} / \bar{\xi}\right)\right.$ divergences and a macroscopic large amount of the bosons aggregates around the impurity. For a finite system, this signals a collapse of the condensate onto the impurity. Due to those effects, we restrict our analysis of the attractive side to moderate values of $\left|g_{\mathrm{IB}}\right|$.

In Fig. 1, mean-field predictions for condensate density and phase are shown for different interaction strengths and a slowly moving impurity. From the analytical solution, we
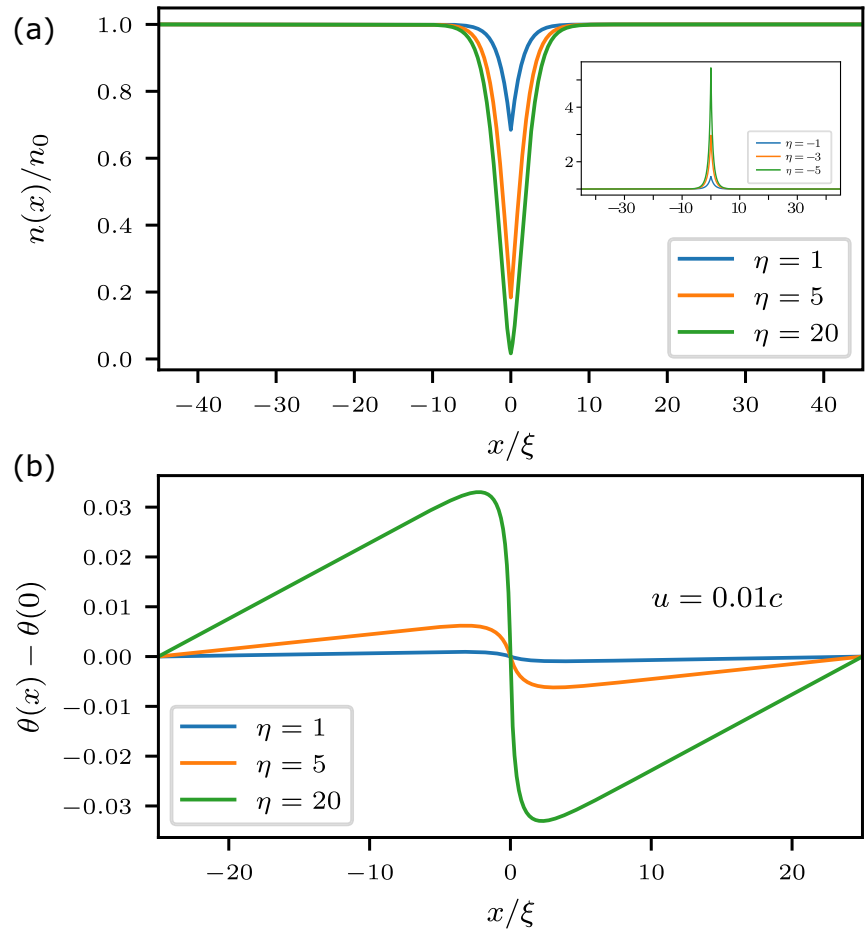

FIG. 1. Mean-field solution for different interactions and various couplings. All other parameters are as in Ref. [15], i.e., $M / m=0.47$, the peak density $n_{0}=7 / \mu \mathrm{m}$, and $g_{\mathrm{BB}}=2.36 \times 10^{-37} \mathrm{Jm}$. For the phase, we fixed $u=0.01 c$, which fixes the total momentum on the mean-field level.

can derive a parameter characterizing the relative condensate deformation,

$$
\eta / n_{0} \bar{\xi}=\eta \sqrt{2 \bar{\gamma}}
$$

with $\bar{\gamma}=\gamma m_{r} / m$, where $\gamma=1 /\left(2 n_{0}^{2} \xi^{2}\right)$ is the so-called Tonks parameter of the 1D Bose gas $[48,49]$, which should be less than unity for the Bogoliubov approximation to hold. The deformation becomes sizable if $\eta / n_{0} \bar{\xi} \sim 1$.

With the analytical expressions for the condensate density and phase, we can calculate the polaron energy $E_{p}=$ $E\left(g_{\mathrm{IB}}\right)-E\left(g_{\mathrm{IB}}=0\right)$ and the effective mass $m^{*}$ of the polaron using $M / m^{*}=\lim _{p \rightarrow 0}\left(1-\frac{P_{\mathrm{B}}}{p}\right)$, with $P_{\mathrm{B}}$ being the mean-field momentum of the condensate, see Ref. [31]. This gives

$$
E_{\mathrm{p}}^{\mathrm{r}, \mathrm{a}}=g_{\mathrm{IB}} n_{0}\left(\frac{|y| \mp 1}{|y| \pm 1}\right)^{2}+\frac{8}{3} n_{0} \bar{c}\left(\frac{3|y| \pm 1}{(|y| \pm 1)^{3}}\right)
$$

for the energies of the repulsive $\left(E_{p}^{(r)}\right.$, upper sign) and attractive $\left(E_{p}^{(a)}\right.$, lower sign) polaron, and for the mass:

$$
\frac{M}{m^{*}}=\frac{M\left(y^{2}-1\right)}{8 n_{0} \bar{\xi} m_{\mathrm{r}} \sqrt{2}+M\left(y^{2}-1\right)} .
$$

These expressions agree with previous findings in Refs. [43,45]. It is interesting to note that for $\eta \rightarrow \infty$, Eq. (8) approaches the energy of a dark soliton and the effective mass $m^{*}$ goes to infinity which is in contrast to results from the extended Fröhlich Hamiltonian [23]. At this point, we note that on the attractive side the solution will collapse to a multiparticle bound state for $\eta \gg 1$, which can be easily seen by noting $E_{\mathrm{p}}^{\mathrm{a}} \rightarrow-\infty$ for $\eta \rightarrow-\infty$. 


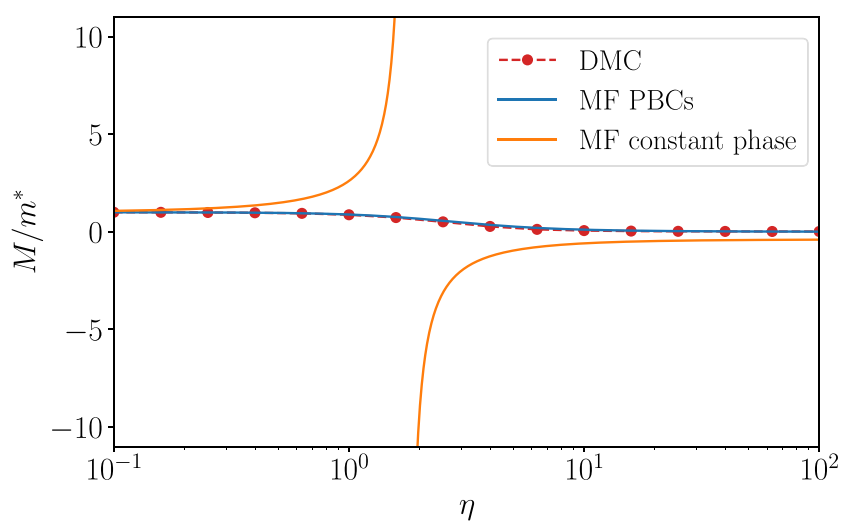

FIG. 2. Polaron mass calculated in mean-field approximation with periodic boundary conditions (PBCs) or constant phase far away from the impurity compared to DMC calculated in Ref. [23]. While the solution using periodic boundary conditions agrees very well with the DMC results, the constant phase solution (or nonperiodic boundary conditions) yields a nonsensical result.

\section{B. Boundary conditions}

We are now going to address the aforementioned importance of the periodic boundary conditions for correctly calculating the effective mass. When imposing periodic boundary conditions, one finds, unsurprisingly, a constant density far away from the impurity, but in contrast the phase is linearly changing at the order of $1 / L$ and therefore not constant. One might be tempted to use a solution where both density and phase are truly constant far away from the impurity (up to exponentially small corrections). A solution with this different boundary condition would still be given by Eqs. (A2) and (A4), but with $\theta_{1}(x)=0$. The effective mass can then be deduced from the wave function in the same fashion as was done for periodic boundary conditions and is plotted in Fig. 2. Calculating the effective mass in this manner, one finds that the effective mass decreases for increasing $\eta$ and it can even become negative. This unphysical result is in clear disagreement with DMC results. Besides that, it also contains a phase jump at infinity which introduces a source term there, which is nonsensical. Addressing this issue from a more technical point of view, it becomes apparent that, strictly speaking, functional derivatives cannot be taken for the constant-phase solutions.
On a mean-field level, this can be alleviated by modifying the functional derivatives by exactly this source term, as has been done in the context of solitons [50,51]. Another possible way to deal with the phase issue is to integrate the phaseout, as has been done in Ref. [45]. Upon considering quantum fluctuations on top of the mean-field solution, none of the above-mentioned methods allow a straightforward generalization. We found expanding about a periodic mean-field solution to be indispensable for the Bogoliubov theory.

We note that this issue persists when extracting the mass from the total momentum dependence of the mean-field energy of the system. That is, when enforcing the nonperiodic phase and expanding the total mean-field energy to quadratic order in the total momentum as $E \approx E_{0}+\frac{p^{2}}{2 m^{*}}$, one obtains an incorrect result for the polaron mass $m^{*}$. On the other hand, when extracting the polaron mass from expanding in $u$ as $E \approx E_{0}+\frac{1}{2} m^{*} u^{2}$, one fortuitously obtains the correct result with both periodic and nonperiodic [44] mean-field solutions.

These difficulties can be traced to the fact that without the phase correction, the mean-field equations of motion do not form a Hamiltonian system. For the full quantum system, one can deduce that the fundamental relation

$$
\frac{d E}{d p}=u
$$

holds exactly by the Feynman-Hellman theorem. Incidentally, this relation can be used to obtain $M / m^{*}=\lim _{p \rightarrow 0}\left(1-\frac{P_{\mathrm{B}}}{p}\right)$, which is used routinely to compute the polaron mass. With periodic boundary conditions, one retains the exact relation Eq. (10) within mean-field theory. On the other hand, when the nonperiodic solution is used, a short calculation gives the relation

$$
\frac{d E_{\mathrm{np}}}{d p}=u-u \bar{n} \frac{d}{d p} \Delta \theta,
$$

where $E_{\mathrm{np}}$ is the total mean-field energy of the nonperiodic state, $\bar{n}$ is the average density, and $\Delta \theta$ is the phase change across the condensate.

\section{QUANTUM FLUCTUATIONS}

After expanding the fields in $\hat{\mathcal{H}}_{\mathrm{LLP}}^{\mathrm{S}}$ in the quantum fluctuations, we find up to second order in $\hat{\xi}^{\dagger}(x)$ and $\delta \hat{u}$

$$
\begin{aligned}
\hat{\mathcal{H}}_{\mathrm{LLP}}^{\mathrm{S}}= & \int d x\left[\hat{\xi}^{\dagger}(x)\left(-\frac{1}{2 m_{\mathrm{r}}} \partial_{x}^{2}+2 g_{\mathrm{BB}}|\phi(x)|^{2}-\mu+g_{\mathrm{IB}} \delta(x)+i u \partial_{x}\right) \hat{\xi}(x)+\frac{g_{\mathrm{BB}}}{2}\left(\phi(x)^{2} \hat{\xi}^{\dagger}(x)^{2}+\text { H.a. }\right)\right] \\
& -i \delta \hat{u} \int \mathrm{d} x\left(\hat{\xi}^{\dagger}(x) \partial_{x} \phi(x)+\phi^{*}(x) \partial_{x} \hat{\xi}(x)\right)-\frac{1}{2} M \delta \hat{u}^{2}
\end{aligned}
$$

with $M \delta \hat{u}=-i \int\left[\phi^{*}(x) \partial_{x} \hat{\xi}(x)+\hat{\xi}^{\dagger}(x) \partial_{x} \phi(x)\right] \mathrm{d} x+\mathcal{O}\left(\hat{\xi}(x)^{2}\right)$, which can be diagonalized by a Bogoliubov rotation to a generalized basis of phonons on a deformed background. We note that for distances far away from the impurity, i.e., $|x| \rightarrow \infty$, these phonons look like the ones of a homogeneous BEC. This allows us to extract the quantum depletion (see Ref. [52] for a detailed discussion on how to regularise the arising UV divergences of the zero point energy). We find for the quantum-corrected density far away from the impurity $n_{0}=n_{0}^{\mathrm{MF}}+\frac{1}{\pi} \sqrt{m_{\mathrm{r}} g_{\mathrm{BB}} n_{0}^{\mathrm{MF}}}$ and thus we have to adjust the mean-field density accordingly. To diagonalize Eq. (12), we note that all terms involving $\delta \hat{u}$ become nonlocal and thus difficult to handle in general, except for the special case $p=$ 0 . This enables us to diagonalize Eq. (12) and to calculate the polaron energy for $p=0$. For a moving impurity, we introduce an approximation setting $\delta \hat{u}=0$ and keep $u$ as 

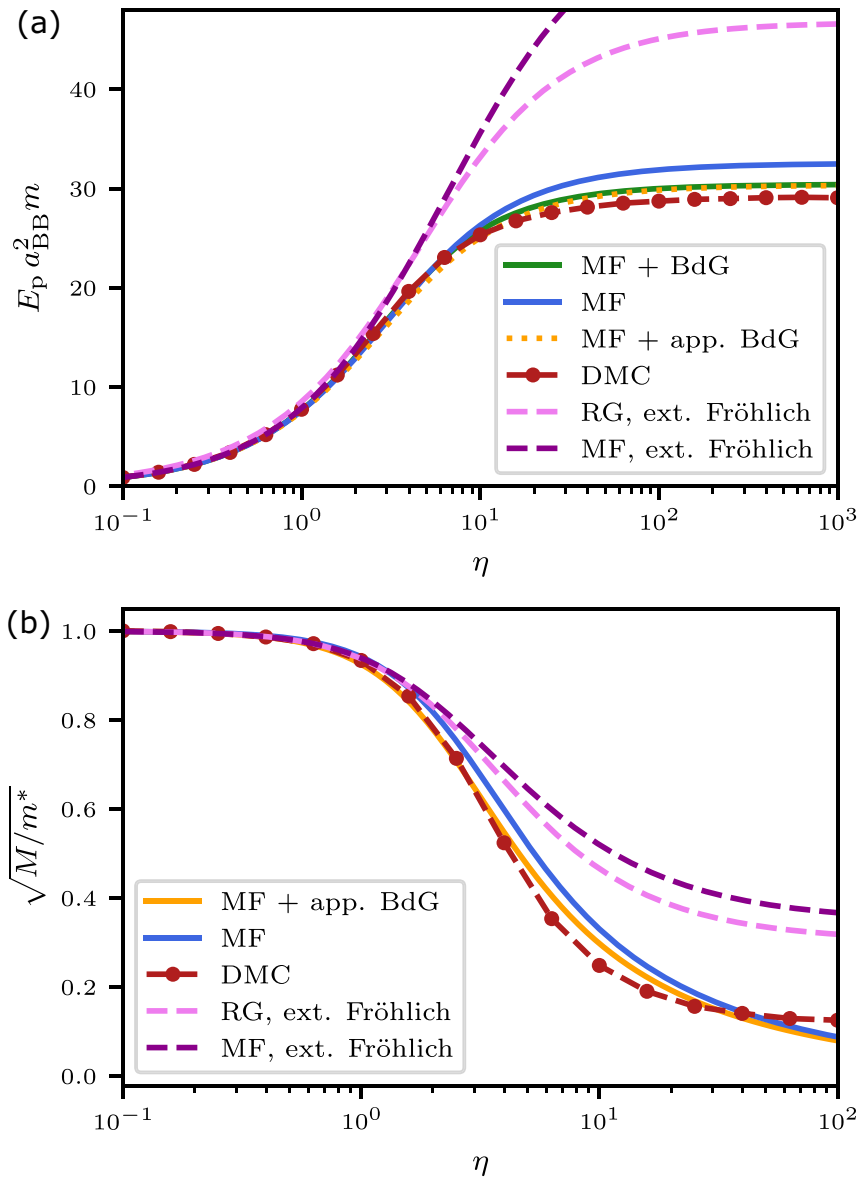

FIG. 3. Polaron energy (a) and effective mass (b) for the repulsive polaron. The curves are obtained using different theoretical methods; all parameters are as in Ref. [15], where $\gamma \approx 0.438$. The DMC, RG, and MF (both based on the extended Fröhlich model) curves were calculated in Ref. [23]. We find exceptional agreement with the DMC results for the energy as well as the effective mass when expanding around the right mean-field solutions and including quantum fluctuations. Only for the very strong coupling regime we do not predict a saturation of the effective mass. The condensate deformation becomes relevant for $\eta / n_{0} \bar{\xi}>1$ Eq. (7), corresponding here to $\eta>1.9$, where predictions from the extended Fröhlich model start to deviate from the full model.

a variational parameter in the mean-field equations. After diagonalizing the remaining quadratic Hamiltonian Eq. (12), $u$ is determined self-consistently,

$$
\begin{aligned}
M u & =P-\left\langle\hat{P}_{\mathrm{B}}\right\rangle, \\
\left\langle\hat{P}_{\mathrm{B}}\right\rangle & =-i \int \phi^{*}(x) \partial_{x} \phi(x) \mathrm{d} x-i\left\langle\int \hat{\xi}^{\dagger}(x) \partial_{x} \hat{\xi}(x) \mathrm{d} x\right\rangle,
\end{aligned}
$$

where the expectation value is taken with respect to the phonon vacuum. For a more detailed description, we refer to Appendix B. Then it is straightforward to calculate the effective mass including the quantum corrections $M / m^{*}=$ $M u / p$. As can be seen in Fig. 3(a), where the energies of the full and approximate solution of the $\mathrm{BdG}$ equations are shown, the approximate treatment of the Hubbard-Stratonovich field is very good.

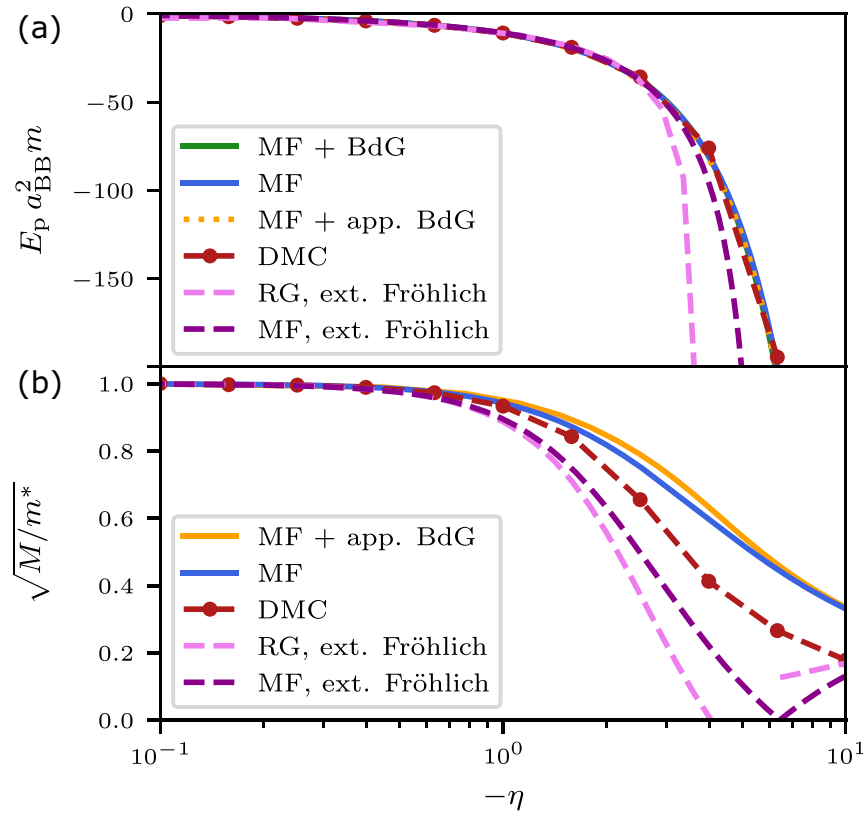

FIG. 4. Polaron energy (a) and mass (b) calculated using different methods for attractive impurity couplings. All parameters are as in Ref. [15] and the RG, MF (based on the extended Fröhlich model), and DMC curves were calculated in Ref. [23]. For the polaron energy, a surprisingly good agreement is achieved with the DMC results, while the agreement is less good for the mass. We explain this by the collapse of the solution to a multiparticle bound state, where we do not expect the mean-field solution to be a good approximation. Furthermore, we do not observe the transition from the attractive to the repulsive polaron observed in the Fröhlich model, signaled by the breakdown of the RG treatment. For more detail on this transition, we refer to Refs. [23,24].

\section{DISCUSSION AND SUMMARY}

Figure 3 shows that already the mean-field solution improves the agreement with DMC simulations significantly for $g_{\mathrm{IB}}>0$ as compared to the Fröhlich model. Including quantum fluctuations leads to almost perfect agreement for the energy. We find, however, that the effective mass diverges for $g_{\text {IB }} \rightarrow \infty$, even after including quantum fluctuations, which seem to be in contrast to the DMC results [23]. This divergence is a characteristic of the 1D geometry and is, for example, also observed in the Tonks limit [34]. One would naively expect this to happen since, for $\eta \gg 1$, the condensate is split into two halves by the impurity, preventing any transport of the condensate across it. The only possible contribution could come from tunneling which is highly suppressed for $\eta \gg 1$. The same reasoning explains why the quantum correction to the effective mass is most significant for intermediate couplings since here the classical current is reduced by the strong condensate deformation, but tunneling is still relevant. The question whether the effective mass actually saturates remains open and other approaches such as DMRG could shed more light on this. As shown in Fig. 4(a) the prediction for the polaron energy is also in very good agreement with DMC data for $g_{\text {IB }}<0$. While qualitatively less accurate for the mass, our approach is free of divergences as compared to the extended Fröhlich model [Fig. 4(b)]. Note that these arguments rely on treating the system as one-dimensional. For experimental 
systems in the one-dimensional regime, we expect that transverse modes may become important for the limiting behavior of $M / \mathrm{m}^{*}$. This analysis has to be done on a case to case basis and we want to stress that all our calculations are benchmarked against strict 1D numerical quantum Monte Carlo results. For a detailed discussion on the influence of the transverse mode and when it's admissible to treat the system investigated in Ref. [15] as strictly one-dimensional, we refer to the detailed discussion in Ref. [23]. Another quantity of experimental relevance [15] is the axial width of the polaron $\left(\left\langle\hat{X}^{2}\right\rangle-\langle\hat{X}\rangle^{2}\right)^{1 / 2}$. In the present paper, which is carried out in the LLP frame and requires translational invariance, such a quantity is infinite. Including a trap potential for the impurity is beyond the scope of the present paper, but could be addressed by using a variational ansatz that is a superposition of ground states (of the infinite system) with different total momenta. On the other hand, studies that do not invoke the LLP transformation can lead to symmetry-broken mean-field states with finite values of the axial width $[36,38]$ even without a trap, but these neglect impurity-BEC entanglement.

In summary, we have shown that a nonperturbative description of the Bose polaron in $1 \mathrm{D}$ requires taking into account the backaction to the condensate while keeping the impurityBEC entanglement. Since the density of phonons defined on such a deformed background remains small, their intrinsic interactions can be neglected to good approximation. Our approach provides a quantitatively accurate and, to a large extent, analytical description of Bose polarons even for strong impurity-boson interactions as long as the boson-boson interactions remain weak. Those findings suggest that a similar method could be used to gain more insight into the polaron formation following a sudden quench. We expect that it will also allow a good description in 3D at and beyond the critical strength of the impurity-boson interaction for self-trapping.

\section{ACKNOWLEDGMENTS}

We would like to thank G. Astrakharchik and J. Brand for useful discussions and insights. We also thank G. Astrakharchik and E. Demler for providing the DMC and RG data of Ref. [23]. M.W. and M.F. acknowledge financial support by the DFG through the SFB/TR 185, Project No. 277625399. J.J. is grateful for support from EPSRC under Grant No. EP/R513052/1. R.B. is grateful for support from a Cecilia Tanner Research Impulse Grant and the Aspen Center for Physics, which is supported by NSF No. PHY1607611 and the Simons Foundation.

\section{APPENDIX A}

In this Appendix, we provide some details on the solution of the mean-field equations used in the main text. The meanfield equations that need to be solved are

$$
\begin{gathered}
\left(-\frac{1}{2 m_{\mathrm{red}}} \partial_{x}^{2}+g_{\mathrm{BB}}|\phi(x)|^{2}-\mu+i u \partial_{x}\right) \phi(x)=0, \\
\left.\partial_{x} \phi(x)\right|_{0-} ^{0+}=2 m_{\mathrm{red}} g_{\mathrm{IB}} \phi(0), \\
\phi(L / 2)=\phi(-L / 2), \\
M u=p-P_{\mathrm{B}} .
\end{gathered}
$$

If we do not require periodic boundary conditions, analytical solutions of the form $|\phi(x)| e^{i \theta_{0}(x)}$ can be found in the literature $[42,47]$. To make use of those solutions, we make the following ansatz:

$$
\phi(x)=\tilde{\phi}(x) e^{i \theta_{1}(x)},
$$

where we introduced $\theta_{1}(x)$, which will be of $\mathcal{O}(x / L)$ and is fixed later on to ensure periodicity of the phase for the meanfield solutions, giving the overall phase $\theta(x)=\theta_{0}(x)+\theta_{1}(x)$. Upon inserting our ansatz into Eq. (A1), we arrive at

$$
\begin{aligned}
& \left(-\frac{1}{2 m_{\mathrm{red}}} \partial_{x}^{2}+g_{\mathrm{BB}}|\tilde{\phi}(x)|^{2}-\tilde{\mu}+i \tilde{u} \partial_{x}\right) \tilde{\phi}(x)=0, \\
& \left.\partial_{x} \tilde{\phi}(x)\right|_{0-} ^{0+}=2 m_{\mathrm{red}} g_{\mathrm{IB}} \tilde{\phi}(0), \\
& e^{i \theta_{1}(L)} \tilde{\phi}(L / 2)=\tilde{\phi}(-L / 2),
\end{aligned}
$$

with the redefinitions $\tilde{\mu}=\mu+\left(\partial_{x} \theta_{1}\right) u / M+\mathcal{O}\left(1 / L^{2}\right)$ and $\tilde{u}=u-\left(\partial_{x} \theta_{1}\right) /\left(m_{\mathrm{r}}\right)$. The solution for this problem is now given by $[42,47]$

$$
\begin{aligned}
|\tilde{\phi}(x)| & =\sqrt{\mu / g_{\mathrm{BB}}}\left(1-\beta \operatorname{sech}^{2}\left(\sqrt{\beta / 2}\left(|x|+x_{0}\right) / \bar{\xi}\right)\right)^{1 / 2}, \\
\theta_{0}(x) & = \begin{cases}f(x) & x>0 \\
2 f(0)-f(-x) & x<0,\end{cases} \\
f(x) & =\arctan \left(\frac{\sqrt{4 u^{2} \beta / \bar{c}^{2}}}{e^{\sqrt{2 \beta}\left(x+x_{0}\right) / \bar{\xi}}-2 \beta+1}\right),
\end{aligned}
$$

with $\quad \beta=1-\frac{u^{2}}{\bar{c}^{2}}+\mathcal{O}\left(1 / L^{2}\right) \quad$ and $\quad \mu=g_{\mathrm{BB}} n_{0}^{\mathrm{MF}}-$ $\left(\partial_{x} \theta_{1}(x)\right) u / M+\mathcal{O}\left(1 / L^{2}\right)$. The jump condition determines $x_{0}$ through a polynomial of order three, but only one solution is stable. It is possible to extract quantities like the critical momentum herein; for a detailed discussion of this, we refer to Ref. [42]. For finite momentum, the condition for $x_{0}$ has to be solved numerically but in the limit $p \rightarrow 0$ we can find the analytical solutions stated in the paper. If we consider the mean-field solution alone and require the number of condensed particles $N$ to stay constant on the mean-field level, we fix $n_{0}^{\mathrm{MF}}=n_{0}\left[1+2 \sqrt{2 \beta} \bar{\xi} / L\left(1-\tanh \left(\sqrt{\beta / 2} x_{0} / \bar{\xi}\right)\right)\right]+\mathcal{O}\left(1 / L^{2}\right)$. Lastly, we fix $\theta_{1}(x)$ to ensure the periodicity of the phase by

$$
\theta_{1}(x)=2[f(0)-f(L / 2)] \frac{x}{L} .
$$

At this point, we note that the $1 / L$ corrections are indeed important when calculating physical quantities. This can be seen by considering the Boson momentum $P_{\mathrm{B}}=$ $\int n(x) \partial_{x} \theta(x) \mathrm{d} x=\int n(x) \partial_{x} \theta_{0}(x) \mathrm{d} x+n_{0}[2(f(0)-f(L / 2)]$. From there we can derive the expressions for $m^{*}$ and $E_{p}$ given in the main text, which are both defined in the limit $p \rightarrow 0$, which allows us to state them fully analytically.

\section{APPENDIX B}

In the following, we give a short overview of the methods used to obtain the quantum corrections to the mean-field solutions. The major steps have been outlined in the main text, and thus we focus on the numerical details. An extensive overview of the techniques used here can be found in Ref. [53]. We note that this is equivalent to solving the resulting Bogolibouv-de Gennes equations. We start by discretizing $\hat{\mathcal{H}}_{\text {LLP }}^{\mathrm{S}}$ from the 
main text after either making the approximation of treating $u$ as a variational parameter or for $p=0$ integrating out the $\hat{u}$-field. For all numerical results presented here, the discretization was done in real space and is therefore straightforward apart from the delta distribution, which was approximated by a Kronecker delta in the following way: $\delta(x) \rightarrow \delta_{i, 0} / a$, where $a$ is the discretization. This comes at the expense of not accounting correctly for the UV behavior. The deviation from the continuum UV behavior is due to discretizing the derivative operators. Nevertheless, for the observables we are interested in here, the UV behavior is not essential, and we found fast convergence; thus, the diagonalization in real space is justified. For notational convenience, we omit the hats on all discretized operators. After discretization, the Hamiltonian can be written as

$$
\begin{aligned}
H_{\mathrm{LLP}}^{\mathrm{S}} & =\sum_{i j}\left[A_{i j} \phi_{i}^{\dagger} \phi_{j}+\frac{1}{2}\left(B_{i j} \phi_{i}^{\dagger} \phi_{j}^{\dagger}+B_{i j}^{*} \phi_{i} \phi_{j}\right)\right] \\
& =\frac{1}{2} \boldsymbol{\Phi}^{\dagger} M \boldsymbol{\Phi}-\frac{1}{2} \operatorname{Tr}(A),
\end{aligned}
$$

where $\boldsymbol{\Phi}^{\dagger}=\left[\phi_{1}^{\dagger}, \phi_{2}^{\dagger}, \ldots \phi_{n}^{\dagger}, \phi_{1}, \phi_{2}, \ldots \phi_{n}\right]$ is the discrete version of $\hat{\xi}(x)$ and $M$ is the semipositive definite matrix

$$
M=\left[\begin{array}{cc}
A & B \\
B^{*} & A^{*}
\end{array}\right] .
$$

At this point, we already note that the trace term is of fundamental importance in 1D since it renders results like the zero-point energy finite without performing additional regularization. Following the steps outlined in Ref. [53], we now diagonalize

$$
v M=\left[\begin{array}{cc}
A & B \\
-B^{*} & -A^{*}
\end{array}\right],
$$

and thus find $T$ such that $T^{\dagger} M T=\operatorname{diag}\left(\omega_{1}, \omega_{2}, \ldots\right.$, $\left.\omega_{n}, \omega_{1}, \omega_{2}, \ldots, \omega_{n}\right)$, while guaranteeing $T^{\dagger} \nu T=v$, which allows us to introduce new bosonic operators

$$
\begin{gathered}
\boldsymbol{\Psi}^{\dagger}=\left[b_{1}^{\dagger}, b_{2}^{\dagger}, \ldots b_{n}^{\dagger}, b_{1}, b_{2}, \ldots b_{n}\right] \text { through } \\
\boldsymbol{\Phi}=T \boldsymbol{\Psi},
\end{gathered}
$$

for which the Hamiltonian takes diagonal form. The new operators $b_{i}$ can be interpreted as quasiparticlelike bosonic excitations with eigenenergy $\omega_{i}$. For a stable polaron, the energy of those excitations is minimized, i.e., the system is in its vacuum state $|0\rangle$ with respect to the $b_{i}$. From here it is then easy to verify that the quantum corrections to the expectation value of an observable of the form $O_{Q}=\sum_{i j} O_{i j} \phi_{i}^{\dagger} \phi_{j}$ is

$$
\begin{aligned}
\left\langle O_{Q}\right\rangle & =\left\langle 0\left|\boldsymbol{\Psi}^{\dagger} T^{\dagger}\left[\begin{array}{ll}
O & 0 \\
0 & 0
\end{array}\right] T \boldsymbol{\Psi}\right| 0\right\rangle \\
& =\left\langle 0\left|\boldsymbol{\Psi}^{\dagger}\left[\begin{array}{ll}
C & D \\
E & F
\end{array}\right] \boldsymbol{\Psi}\right| 0\right\rangle=\operatorname{Tr}(F) .
\end{aligned}
$$

To conclude this section, we will comment on the IR (infrared) divergences that are characteristic in 1D systems and how they are dealt with here. First, we note that quantities like the twopoint function

$$
\left\langle\phi_{i}^{\dagger} \phi_{i}\right\rangle=\left\langle 0\left|\left(\boldsymbol{\Psi}^{\dagger} T^{\dagger}\right)_{i}(T \boldsymbol{\Psi})_{i}\right| 0\right\rangle \sim L
$$

are indeed IR divergent in our treatment. For the global quantities and $p=0$, this can be dealt with as outlined in the main text by considering the zero-point energy,

$$
E=\frac{1}{2}\left(\sum_{i} \omega_{i}-\operatorname{Tr}(A)\right),
$$

which is UV and IR finite and then taking adequate derivatives (i.e., with respect to the chemical potential for the depletion). When considering $\hat{\mathcal{H}}_{\mathrm{LLP}}^{\mathrm{S}}$ for $p \neq 0$ without any approximations, the phonon momentum seems to be IR divergent and also, for the polaron energy, we found a system-size dependence. Lastly, we remark that in the approximate treatment, i.e., when viewing $u$ as a variational parameter, the phonon momentum remains IR and UV finite. Therefore, we conclude that all results presented in the main text are cutoff independent, and no divergences occur.
[1] L. Landau, Phys. Z. Sowjetunion 3, 644 (1933).

[2] L. D. L. S. I. Pekar, Zh. Eksp. Teor. Fiz. 16, 335 (1946).

[3] N. Mott, Phys. C Supercond. 205, 191 (1993).

[4] A. S. Alexandrov and J. T. Devreese, Advances in Polaron Physics (Springer-Verlag, Berlin, 2010), Vol. 159.

[5] H. Bässler and A. Köhler, Top. Curr. Chem. 312, 1 (2012).

[6] A. Schirotzek, C. H. Wu, A. Sommer, and M. W. Zwierlein, Phys. Rev. Lett. 102, 230402 (2009).

[7] Y. Zhang, W. Ong, I. Arakelyan, and J. E. Thomas, Phys. Rev. Lett. 108, 235302 (2012).

[8] C. Kohstall, M. Zaccanti, M. Jag, A. Trenkwalder, P. Massignan, G. M. Bruun, F. Schreck, and R. Grimm, Nature 485, 615 (2012).

[9] M. Koschorreck, D. Pertot, E. Vogt, B. Fröhlich, M. Feld, and M. Köhl, Nature 485, 619 (2012).

[10] F. Scazza, G. Valtolina, P. Massignan, A. Recati, A. Amico, A. Burchianti, C. Fort, M. Inguscio, M. Zaccanti, and G. Roati, Phys. Rev. Lett. 118, 083602 (2017).
[11] M. Cetina, M. Jag, R. S. Lous, J. T. Walraven, R. Grimm, R. S. Christensen, and G. M. Bruun, Phys. Rev. Lett. 115, 135302 (2015).

[12] M. Cetina, M. Jag, R. S. Lous, I. Fritsche, J. T. Walraven, R. Grimm, J. Levinsen, M. M. Parish, R. Schmidt, M. Knap, and E. Demler, Science 354, 96 (2016).

[13] M. Parish and J. Levinsen, Phys. Rev. B 94, 184303 (2016).

[14] P. Massignan, M. Zaccanti, and G. M. Bruun, Rep. Prog. Phys. 77, 034401 (2014).

[15] J. Catani, G. Lamporesi, D. Naik, M. Gring, M. Inguscio, F. Minardi, A. Kantian, and T. Giamarchi, Phys. Rev. A 85, 023623 (2012).

[16] N. B. Jørgensen, L. Wacker, K. T. Skalmstang, M. M. Parish, J. Levinsen, R. S. Christensen, G. M. Bruun, and J. J. Arlt, Phys. Rev. Lett. 117, 055302 (2016).

[17] M. G. Hu, M. J. Van De Graaff, D. Kedar, J. P. Corson, E. A. Cornell, and D. S. Jin, Phys. Rev. Lett. 117, 055301 (2016). 
[18] Z. Z. Yan, Y. Ni, C. Robens, and M. W. Zwierlein, Science 368, 190 (2020).

[19] S. P. Rath and R. Schmidt, Phys. Rev. A 88, 053632 (2013).

[20] G. E. Astrakharchik and L. P. Pitaevskii, Phys. Rev. A 70, 013608 (2004).

[21] J. Tempere, W. Casteels, M. K. Oberthaler, S. Knoop, E. Timmermans, and J. T. Devreese, Phys. Rev. B 80, 184504 (2009).

[22] W. Casteels, T. Van Cauteren, J. Tempere, and J. T. Devreese, Laser Phys. 21, 1480 (2011).

[23] F. Grusdt, G. E. Astrakharchik, and E. Demler, New J. Phys. 19, 103035 (2017).

[24] Y. E. Shchadilova, R. Schmidt, F. Grusdt, and E. Demler, Phys. Rev. Lett. 117, 113002 (2016).

[25] H. Fröhlich, Adv. Phys. 3, 325 (1954).

[26] W. Li and S. Das Sarma, Phys. Rev. A: At. Mol. Opt. Phys. 90, 013618 (2014).

[27] J. Levinsen, M. M. Parish, and G. M. Bruun, Phys. Rev. Lett. 115, 125302 (2015).

[28] W. Casteels and M. Wouters, Phys. Rev. A: At. Mol. Opt. Phys. 90, 043602 (2014).

[29] R. S. Christensen, J. Levinsen, and G. M. Bruun, Phys. Rev. Lett. 115, 160401 (2015).

[30] T. Ichmoukhamedov and J. Tempere, Phys. Rev. A 100, 043605 (2019).

[31] F. Grusdt and E. Demler, arXiv:1510.04934.

[32] A. Lampo, C. Charalambous, M. Á. García-March, and M. Lewenstein, Phys. Rev. A 98, 063630 (2018).

[33] L. A. Pena Ardila and S. Giorgini, Phys. Rev. A 92, 033612 (2015).

[34] L. Parisi and S. Giorgini, Phys. Rev. A 95, 023619 (2017).
[35] J. P. Hernandez, Rev. Mod. Phys. 63, 675 (1991).

[36] F. M. Cucchietti and E. Timmermans, Phys. Rev. Lett. 96, 210401 (2006).

[37] M. Bruderer, W. Bao, and D. Jaksch, Europhys. Lett. 82, 30004 (2008).

[38] A. A. Blinova, M. G. Boshier, and E. Timmermans, Phys. Rev. A 88, 053610 (2013).

[39] T. D. Lee, F. E. Low, and D. Pines, Phys. Rev. 90, 297 (1953).

[40] A. Kantian, U. Schollwöck, and T. Giamarchi, Phys. Rev. Lett. 113, 070601 (2014).

[41] T. Lausch, A. Widera, and M. Fleischhauer, Phys. Rev. A 97, 033620 (2018).

[42] V. Hakim, Phys. Rev. E 55, 2835 (1997).

[43] A. G. Volosniev and H. W. Hammer, Phys. Rev. A 96, 031601(R) (2017).

[44] S. I. Mistakidis, A. G. Volosniev, N. T. Zinner, and P. Schmelcher, Phys. Rev. A 100, 013619 (2019).

[45] G. Panochko and V. Pastukhov, Ann. Phys. (NY) 409 (2019).

[46] G. D. Mahan, Many-Particle Physics (Springer, Berlin, 2000).

[47] T. Tsuzuki, J. Low Temp. Phys. 4, 441 (1971).

[48] M. Girardeau, J. Math. Phys. 1, 516 (1960).

[49] E. H. Lieb and W. Liniger, Phys. Rev. 130, 1605 (1963).

[50] I. M. Uzunov and V. S. Gerdjikov, Phys. Rev. A 47, 1582 (1993).

[51] I. V. Barashenkov and E. Y. Panova, Phys. D 69, 114 (1993).

[52] L. Salasnich and F. Toigo, Phys. Rep. 640, 1 (2016).

[53] M.-w. Xiao, arXiv:0908.0787.

Correction: The previously published Figures 1(a) and 1(b) contained plotting errors and have been replaced with the corrected plots. 